\title{
THE UPDATING OF GEOSPATIAL BASE DATA
}

\author{
Muhamad N. Alrajhi ${ }^{1}$ Gottfried Konecny ${ }^{2, *}$ \\ ${ }^{1}$ Ministry of Municipal and Rural Affairs (MOMRA), Riyadh, KSA \\ ${ }^{2}$ Leibniz University Hannover, Germany - konecnygottfried@yahoo.de
}

Commission III, ICWG IV/III

KEY WORDS: Updating, Geospatial Base Data, Saudi Arabia

\begin{abstract}
:
Topopographic mapping issues concern the area coverage at different scales and their age. The age of the map is determined by the system of updating. The United Nations (UNGGIM) have attempted to track the global map coverage at various scale ranges, which has greatly improved in recent decades. However the poor state of updating of base maps is still a gglobal problem. In Saudi Arabia large scale mapping is carried out for all urban, suburban and rural areas by aerial surveys. Updating is carried out by remapping every 5 to 10 years. Due to the rapid urban development this is not satisfactory, but faster update methods are forseen by use of high resolution satellite imagery and the improvement of object oriented geodatabase structures, which will permit to utilize various survey technologies to update the photogrammetry established geodatabases. The longterm goal is to create an geodata infrastructure, which exists in Great Britain or Germany.
\end{abstract}

\section{HISTORY OF LARGE SCALE TOPOGRAPHIC MAPPING}

Geospatial data provision has historically been the task of mapping. A map constitutes a geometric and thematic model of the earth's surface in a suitable projection. A thematic map serves the needs of specific themes of interest to the community. It relies in geometry on a base map, which is common to all themes and has a minimum of features. This is usually a topographic map showing the 3D features of the terrain in a $21 / 2$ $\mathrm{D}$ representation. But also a $2 \mathrm{D}$ cadastral map can serve the function of a base map, if it is based on high geometric standards established by geodetic surveys. Al mapping is scale dependent.

Topographic mapping of entire countries in a systematic manner has been made possible after the geodetic efforts by the Academie Francaise in the 18th century. It was introduced by the „Carte Geometrique de la France in 1745/1748. It consisted of a map at the scale 1:86 400, which was comleted fort he entire territory of France in 1789.

At that time also other European countries made similat efforts, for example Emperor Joseph II of Austria covering his Monarchy by maps 1:28 800. In the Kingdom od Hannover a map at the scale 1:21 333 was compiled between 1764 and 1784 . In Britain a map of Hartfordshire was compiled in 1766 and in India the Survey of India was established in 1767. In the United States of America the US.Congress authorized the Geological Survey in 1884 to start to map the USA at a scale 1:62 500.

Systematic mapping at large scale was initiated in 1817 by the survey of cadastral boundaries displayed in cadastral maps of the Fracisean Cadastre of the entire Austro-Hungarian Monarchy at the scale 1:2880 introduced by Emperor Franz. These topographic and cadastral mapping systems served as the base for the thematic mapping efforts by others at a later date.
By the year 1900 the global mapping extent at medium scales covered most of Europe and parts of India and the USA (see Fig.1)

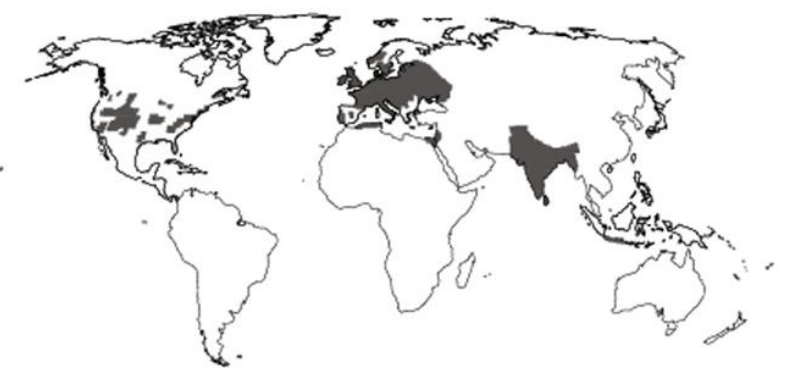

Fig.1 Topographic Mapping Status in the Year 1900

The other regions of the globe needed more efficient survey technologies than terrestrial measurements used at that time to be able to map the vast territories. These came along during World War I , when an aerial survey camera was invented along with an appropriate photogrammetric opto-mechanical restituton technology to transfer stereo images into maps. It took several decades and World War II to prove that photogrammetry was indeed capable to create maps for entire continents. While the war efforts were military, the civilian public use, especially in developing countries, was made possible by the establishment of the International Training Center for Aerial Surveys ITC in Delft, Netherlands in 1951 by the Dutch government as a contribution to the United Nations to make photogrammetric technology globally accessible as a mapping tool.

\section{UNGGIM SURVEYS ON COVERAGE AND AGE OF EXISTING TOPOGRAPHIC MAPS}

The United Nations Secretariat has followed up the global mapping effort by holding United Nations Cartographic Conferences every 3 or 4 years in Asia and in the Americas until in 2012 United Nations Geospatial Information Management UNGGIM was created as a new entitity at the UN 
Secretariat. UNCC and UNGGIM have followed the progress of nowadays digital mapping and the administration of geospatial data in geodatabases at 4 different scale ranges: range I 1:25 000 , range II $1: 50000$, range III 1:100 000 and range IV 1:250 000 for the years 1968, 1974, 1980, 1986 and 2012 (see Fig.2)

\begin{tabular}{|c|c|c|c|c|}
\hline \multicolumn{5}{|l|}{ World summary: } \\
\hline & range I & range II & range III & range IV \\
\hline area of survey 1986 & $90.1 \%$ & $97.4 \%$ & $97.0 \%$ & $97.75 \%$ \\
\hline 1986 map coverage & $17.9 \%$ & $49.3 \%$ & $46.4 \%$ & $87.5 \%$ \\
\hline 1980 map coverage & $13.3 \%$ & $42.2 \%$ & $42.2 \%$ & $80.0 \%$ \\
\hline 1974 map coverage & $11.6 \%$ & $35.0 \%$ & $40.5 \%$ & $80.5 \%$ \\
\hline 1968 map coverage & $7.7 \%$ & $23.4 \%$ & $38.2 \%$ & $81.0 \%$ \\
\hline \multicolumn{5}{|c|}{ Since the last survey in 1986 considerable progress has been made in data coverage: } \\
\hline & range I & range II & range III & range IV \\
\hline 2012 map coverage & $33,5 \%$ & $81.4 \%$ & $67.5 \%$ & $98.4 \%$ \\
\hline
\end{tabular}

Fig. 2 Topographic Mapping Status 1968 to 2015

These surveys confirm, that there has been continuing progress in trying to get a global map coverage. In 2012 it is nearly available at the scale range 1:250 000. At the scale range 1:50 $00081.4 \%$ of the global land area are covered with the exception of the polar regions. At the scale range 1:25 000 only a coverage of $33.5 \%$ exists.

The other important issue is the age of the data content varies; the 1:25 000 scale data are on average 22.4 years old and the 1:50 000 scale data 26.3 years old. The age of map data varies for the different continents, e.g. for 1:50 000 for Africa 35.1 years, for Asia 27.2 years and for Europe 17.1 years (see Fig.3).

No global information is available for the urban scales 1:1000 and 1:5000. But the problems for large scale data coverage exist as they do for medium scales shown above.

Problem 1 is coverage and problem 2 is the update of the coverage.

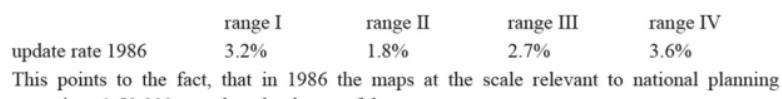

This points to the fact, that in 1986 the maps at the scale relevant to national planning operations 1:50 000 were hopelessly out of date.

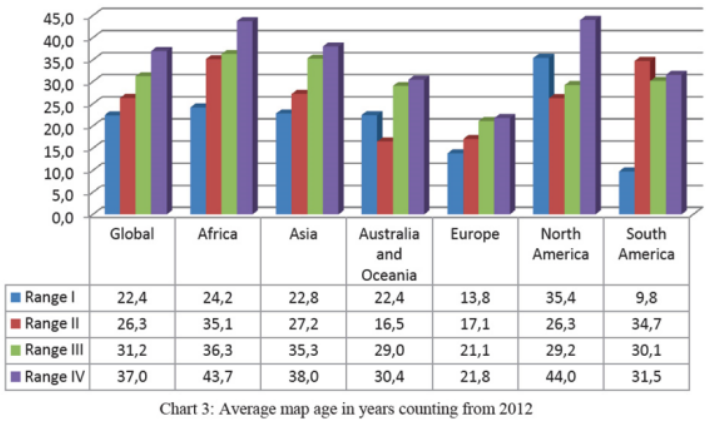

Fig.3 Map Age

\section{THE MAP UPDATING PROBLEM AS EXPERIENCED IN SAUDI ARABIA}

The Ministry of Municipal and Rural Affairs of Saudi Arabia (MOMRA) maintains a huge medium to large scale topographic mapping system as shown in Fig.4 and 5.
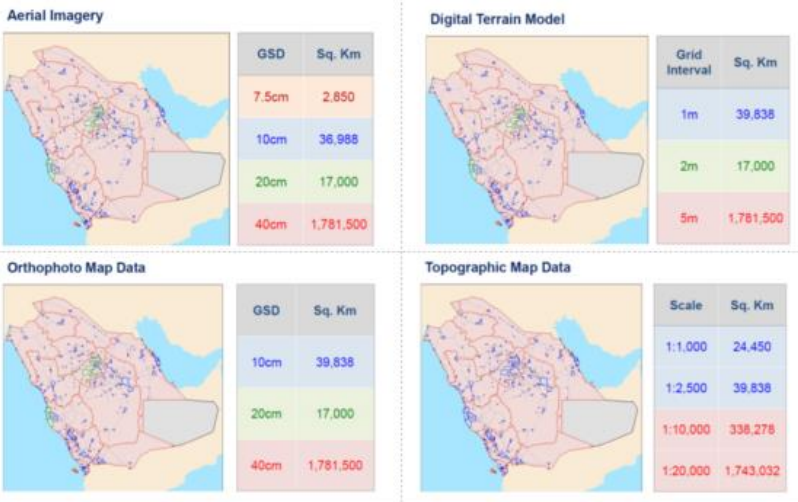

Fig.4 Geospatial Data maintained bv MOMRA
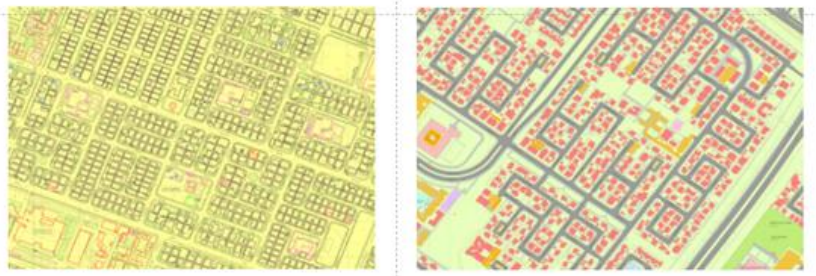

Fig.5 Sample maps 1:1000 in vector and thematic form

To update the geodatabase of digital base maps compiled by photogrammetric mapping three possibilities exist: (see Fig.6)

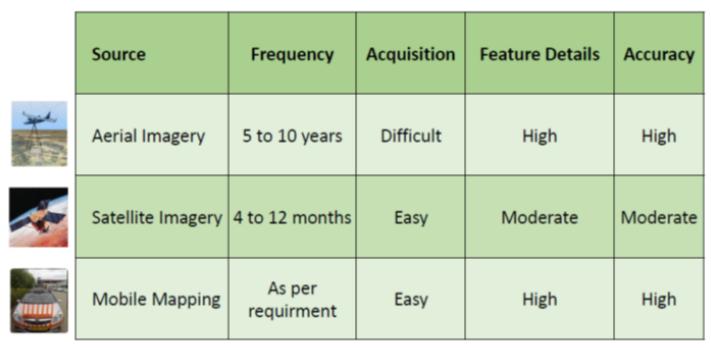

Fig.6 Updating possibilities

1) Updating by using new aerial imagery and by new photogrammetric line mapping is currently carried out. However this is currently not possible faster than every 5 to 10 years für the entire dataset. New mapping areas are simple replaced in the ArcGIS geodatabase with the new data, when reflown and remapped at all scales (e.g.1:1000, 1:10 000)

2) An annual updating cycle for priority areas is made possible from high resolution satellite images with GSD 0.3 to $1 \mathrm{~m}$. For this possibility a separate ArcGIS geodatabase is crated for mappable features comparable to the scale 1:5000

3) Another approach has been suggested using a new street view mobile mapping approach. However there are two handicaps for use of this method: The first is security restrictions by the authorities. But second the updating by a method other than by aerial photography is only possible by use of an updatable object oriented geodatabase, such as Oracle Spatial, and not ArcGIS, which can be slightly modified to an object structure, but not easily for all topographic features. In the longrun the changeover to an object relational database is envisiged, to make use of updating methods other than aerial imagery possible. 
This is particularly desirable, when integrating cadastral data into the geodatabase. Since these require tranaction updates in near real time.

\section{INCORPOATED MODELS FOR A SATISFACTORY GEODATABASE UPDATING SYSTEM}

While only few countries have been covered by systematic large scale surveys, there are only few examples for a satisfactory update methodology: Great Britain and Germany.

Great Britain with a territoty of $219331 \mathrm{~km} 2$ and a population of 60 million is covered by the Ordnance Survey Master Map at scales 1:1250 in urban areas and 1:2500 in rural areas. The Ordnance Survey with a staff of 1190 and an operating cost budget of 83 million British Pounds keeps the topographic data system up to date by help of outsourcing contracts within 6 months.

Germany with a territory of $357386 \mathrm{~km} 2$ and a population of 82 million is covered by cadastral geometric records equivalent to a scale 1:1000 (ALKIS) and topographic records (ATKIS) equivalent to a scale 1:5000. The cadastral records are maintained by 226 survey authorities with a total staff of about 10000 professionals in the 16 states of the Federal Republic on a transaction basis. The updates are available in the system within 1 to 2 months for parcel boundaries and buildings. Other topographic data are updated every 1 to 2 years using photogrammetry or GNSS surveys.

\section{CONCLUSIONS}

Both Britain and Germany possess an efficient, but costly professional infrastructure, which in most other countries of the globe does not exist and cannot be built up in less than a generation.

Therefore most other countries must solve their large scale data problems in other ways.

This is the case for the Kingdom of Saudi Arabia, where topographic maps of urban areas are generated on the basis of international photogrammetry contracts.

Vector maps have been created for $24450 \mathrm{~km} 2$ at the scale 1:1000 (urban areas), for $39838 \mathrm{~km} 2$ at the scale 1:2500 (suburban areas) and for $338278 \mathrm{~km} 2$ at the scale 1:10 000 (rural areas). These vector data are administered in an ArcGIS geodatabase. The mapped areas are reflown by aerial photography every 5 to 7 years. Digital orthophotos created from this imagery then provide a fast record of the changes in topography when superimposed on the vector data in the geodatabase. Then new mapping becomes possible within a 2 year period. Presently $1,781,500 \mathrm{~km} 2$ are covered by orthoimages at $40 \mathrm{~cm} \mathrm{GSD,} \mathrm{17,000} \mathrm{km} 2$ at $20 \mathrm{~cm}$ GSD and 39 $838 \mathrm{~km} 2$ at $10 \mathrm{~cm}$ GSD.

While this scheduling of reflights offers some flexibility to meet needs, the entite remapping at 5 to 7 year intervals is still considered as unsatisfactory, since it does not allow for a continuous update of the database at the scales required.

So that updates of the features surveyed by other technologies than photogrammetry (e.g. GNSS ground surveys) can be made in the geodatabase, this geodatabase must be converted into an object oriented structure. This conversion is currently contemplated.
Another approach is to avoid the time consuming and costly tendering procrdure for aerial flights, since nowadays high resolution satellite imagery with a ground sample distance of up to $30 \mathrm{~cm}$ is becoming available. This resolution limit determines the usable scale for urban geospatial data derived from satellite imagery. Thus a scale limit for these data become 1:5000.

It is therefore contemplated to obtain such satellite imagery of urban centers with a GDS of 30 or $50 \mathrm{~cm}$, preferrably in stereo, at annual intervals and to generate an automated vector map with an object oriented data structure for buildings and roads, which is updatable by GNSS surveys to reach a data system with data not older than 1 year.

It may be, that a system based on the 1:5000 scale with limited feature content falls short of all accuracy requirements inherent to a 1:1000 scale based system used currently, but the capability to continuously update the system within one year is an advantage which should not be overlooked, since the establishment of a system as it is now practised in Great Britain or Germany is currently out of reach, since an equivalent infrastructure cannot be built up in a short time.

\section{REFERENCES}

Alrajhi, M.N., 2013. A Semi-Automated Procedure for Orthophto Generation from High Resolution Satellite Imagery. In: Wissenschaftliche Arbeiten der Fachrichtung Geodäsie und Geoinformatik der Leibniz Universität Hannover, ISSN 01741454, Nr. 307, Dissertation, Hannover.

Alrajhi, M., Alam, Z., Khan, A.M., Alobeid, A., 2016. Influence of GSD for 3D City Modeling and Visualization from Aerial Imagery. In: The International Archives of the Photogrammetry, Remote Sensing and Spatial Information Sciences, Sci. XLI-B3, pp. 561-565.

Alrajhi, M., Janjua, S.K., Khan, A.M., Alobeid, A.,2016. Updating Maps Using High Resolution Satellite Imagery. In: The International Archives of the Photogrammetry, Remote Sensing and Spatial Information Science, Sci. XLI-B4, pp. 711719.

AlObeid, Al Rajhi, M., 2012. Pixel based matching of satellite stereo pairs for build up areas. In: The 35th Plenary and Working Groups Meeting of (ISO/TC 211), Jeddah, Kingdom of Saudi Arabia.

Konecny, G., Breitkopf, U., Radtke, A., 2016. The Status of Topographic Mapping in the World - A UNGGIM-ISPRS Project 2012-2015. In: ZfV 141 (2016), no. 1, pp. 20-26.

Monmonier., 2015. History of Cartography, Volume 6, Cartography in the Twentieth Century. The University of Chicago Press, Chicago 60637, ISBN: 13.978-0-226.534469-5 